\title{
New soliton solutions of anti-self-dual Yang-Mills equations
}

\author{
Masashi Hamanaka and Shan-Chi Huang \\ Department of Mathematics, University of Nagoya, \\ Nagoya, 464-8602, Japan \\ E-mail: hamanaka@math.nagoya-u.ac.jp, x18003x@math.nagoya-u.ac.jp
}

ABSTRACT: We study exact soliton solutions of anti-self-dual Yang-Mills equations for $G=\mathrm{GL}(2)$ in four-dimensional spaces with the Euclidean, Minkowski and Ultrahyperbolic signatures and construct special kinds of one-soliton solutions whose action density $\operatorname{Tr} F_{\mu \nu} F^{\mu \nu}$ can be real-valued. These solitons are shown to be new type of domain walls in four dimension by explicit calculation of the real-valued action density. Our results are successful applications of the Darboux transformation developed by Nimmo, Gilson and Ohta. More surprisingly, integration of these action densities over the four-dimensional spaces are suggested to be not infinity but zero. Furthermore, whether gauge group $G=\mathrm{U}(2)$ can be realized on our solition solutions or not is also discussed on each real space.

KEYwords: Integrable Field Theories, Solitons Monopoles and Instantons, Integrable Hierarchies

ARXIV EPRINT: 2004.09248

Dedicated to the memory of Jon Nimmo 


\section{Contents}

1 Introduction 1

2 Soliton solutions on four-dimensional complex spaces $\quad 2$

2.1 Anti-self-dual Yang-Mills equations 2

2.2 Soliton solutions and action densities for $G=\mathrm{GL}(2)$

3 Soliton solutions on four-dimensional real spaces $\quad 6$

$\begin{array}{lll}3.1 & \text { On Euclidean real space } \mathbb{E} & 7\end{array}$

3.2 On Minkowski Real Space $\mathbb{M} \quad 9$

$\begin{array}{lll}3.3 & \text { On ultrahyperbolic real space } \mathbb{U}_{1} & 10\end{array}$

$\begin{array}{lll}3.4 & \text { On ultrahyperbolic real space } \mathbb{U}_{2} & 11\end{array}$

4 Comparison to already known soliton solutions $\quad 12$

4.1 Atiyah-Ward ansatz solutions $(G=\mathrm{GL}(2)) \quad 12$

4.2 't Hooft ansatz solutions $(G=\mathrm{SU}(2)) \quad 13$

5 Conclusion and discussion $\quad 13$

$\begin{array}{ll}\text { A Calculation of action density (2.12) } & 14\end{array}$

\section{Introduction}

Yang-Mills theories are at the center of elementary particle physics to describe fundamental laws of interactions. Topological solitons in these theories, such as instantons, monopoles, vortices, calorons, merons, played central roles in the study of non-perturbative aspects, duality structures, quark confinements and so on. (See e.g. [1, 5, 8, 10, 13, 16, 24, 26, 29].) To study these topological solitons, the anti-self-dual (ASD) Yang-Mills equation would be in the most important position. For instance, the instantons are global solutions of this equation with a special boundary condition such that the action is finite. For mathematical aspects, the instantons are described very elegantly by the ADHM construction [2].

On the other hand, the anti-self-dual Yang-Mills equation has a very close relationship with lower-dimensional integrable equations, such as the KdV equation, the Toda equations, the Painlevé equations and so on [18, 30]. Energy densities of some soliton solutions to these equations are localized on hyperplanes in the whole space-time dimensions and hence they can be interpreted as domain walls in the space-times. Existence of these solitons solutions also relate to their integrability, such as existence of infinite conserved quantities and existence of hidden infinite symmetries. For anti-self-dual Yang-Mills equations, the domain wall type soliton solutions exist as well and can be constructed from the 
't Hooft ansatz and the Atiyah-Ward ansatz. However, already known soliton solutions given in section 4 always lead to trivial action densities as we will see.

In this paper, we construct exact soliton solutions of anti-self-dual Yang-Mills equations for $G=\mathrm{GL}(2)$ and calculate the action densities of them on four-dimensional real spaces with the Euclidean, Minkowski and Ultrahyperbolic signatures. We find that these type Soliton solutions lead to real-valued action densities which can be interpreted as nontrivial domain walls in four-dimension. This beautiful result is a successful application of the Darboux transformation developed by Nimmo, Gilson and Ohta [21]. More surprisingly, integration of these non-trivial action densities over the four-dimensional spaces are suggested to be not infinity but zero. We also discuss in details whether gauge group could be unitary on our solition solutions or not and find that $G=\mathrm{SU}(2)$ could be realized in one kind of the Ultrahyperbolic signature.

This paper is organized as follows. In section 2, we introduce the anti-self-dual YangMills equations on four-dimensional complex spaces and give exact soliton solutions together with action densities of them. In section 3, we present exact soliton solutions with real valued action densities by taking some dimensional reduction conditions on the complex spaces and discuss the possibility of realization of unitary gauge group on each real space. In section 4, we review some already known soliton solutions of the anti-self-dual Yang-Mills equations and show that they are all trivial in the sense of action density while our solutions are non-trivial. Section 5 is devoted to conclusion and discussion.

\section{Soliton solutions on four-dimensional complex spaces}

In this section, we give a complex version of four-dimensional anti-self-dual Yang-Mills equations which is a unified treatment of section 3. In section 2.1, we introduce a formulation of anti-self-dual Yang-Mills equations on four-dimensional complex spaces which relates to the twistor theory, following the conventions close to that in the book of Mason and Woodhouse [18]. In section 2.2, we calculate a complex-valued action density of exact soliton solutions [11] generated by the Darboux transformation [21]. This complexvalued action density would be reduced to four-dimensional real spaces with three kinds of signatures in section 3 and the reduced action densities could be real-valued by taking some conditions.

\subsection{Anti-self-dual Yang-Mills equations}

Let $(z, \widetilde{z}, w, \widetilde{w})$ be a double null coordinates on four-dimensional complex spaces with metric defined by

$$
\begin{aligned}
d s^{2}=g_{m n} d z^{m} d z^{n}= & 2(d z d \widetilde{z}-d w d \widetilde{w}), \quad m, n=1,2,3,4 . \\
\text { where } g_{m n} & :=\left(\begin{array}{cccc}
0 & 1 & 0 & 0 \\
1 & 0 & 0 & 0 \\
0 & 0 & 0 & -1 \\
0 & 0 & -1 & 0
\end{array}\right),\left(z^{1}, z^{2}, z^{3}, z^{4}\right):=(z, \widetilde{z}, w, \widetilde{w}) .
\end{aligned}
$$


We can recover three kinds of real spaces by taking some suitable reality conditions on $z, \widetilde{z}, w, \widetilde{w}$ as follows. Concrete realizations are given in section 3 .

- Reality condition: $\widetilde{z}=\bar{z}, \widetilde{w}=-\bar{w}$ gives the Euclidean real space $\mathbb{E}$.

- Reality condition: $z, \widetilde{z} \in \mathbb{R}, \widetilde{w}=\bar{w}$ gives the Minkowski real space $\mathbb{M}$.

- Reality condition: $\widetilde{z}=\bar{z}, \widetilde{w}=\bar{w}$ gives the Ultrahyperbolic real space $\mathbb{U}_{1}$.

- Reality condition: $z, \widetilde{z}, w, \widetilde{w} \in \mathbb{R}$ gives the Ultrahyperbolic real space $\mathbb{U}_{2}$.

Note that $\mathbb{U}_{1}$ and $\mathbb{U}_{2}$ are different real slices even though their signature are the same.

Let us consider a gauge theory on the complex space and assume gauge group to be $G=\operatorname{GL}(N)$. The field strengths are defined by

$$
F_{m n}:=\partial_{m} A_{n}-\partial_{n} A_{m}+\left[A_{m}, A_{n}\right]
$$

where $A_{m}(z)$ denote gauge fields which take values in the Lie algebra of $G$. The anti-selfdual Yang-Mills equation on the complex space is defined as follows:

$$
F_{z w}=0, F_{\widetilde{z} \widetilde{w}}=0, F_{z \widetilde{z}}-F_{w \widetilde{w}}=0
$$

which reduces to the standard anti-self-dual Yang-Mills equations on real slices in the sense of Hodge dual as we will see in section 3.

In order to find the solution of the anti-self-dual Yang-Mills equations, let us begin with the Yang equation:

$$
\partial_{\widetilde{z}}\left(J^{-1} \partial_{z} J\right)-\partial_{\widetilde{w}}\left(J^{-1} \partial_{w} J\right)=0,
$$

where the $N \times N$ matrix is called Yang's $J$-matrix. Then ASD gauge fields could be obtained from a solution $J$ of the Yang equation by decomposing $J$ into $N \times N$ two matrices $h$ and $\widetilde{h}$ such that $J=\widetilde{h}^{-1} h,{ }^{1}$ and setting:

$$
A_{z}=-\left(\partial_{z} h\right) h^{-1}, A_{w}=-\left(\partial_{w} h\right) h^{-1}, A_{\widetilde{z}}=-\left(\partial_{\widetilde{z}} \widetilde{h}\right) \widetilde{h}^{-1}, A_{\widetilde{w}}=-\left(\partial_{\widetilde{w}} \widetilde{h}\right) \widetilde{h}^{-1} .
$$

Note that the gauge transformation acts on $h$ and $\widetilde{h}$ as $h \mapsto g h, \widetilde{h} \mapsto g \widetilde{h}, g(x) \in G$ and hence Yang's matrix $J$ is gauge invariant. If we take a special gauge $\widetilde{h}=1$, gauge fields become a simpler form in terms of $J$ :

$$
A_{z}=J^{-1} \partial_{z} J, \quad A_{w}=J^{-1} \partial_{w} J, \quad A_{\widetilde{z}}=A_{\widetilde{w}}=0,
$$

and satisfy the anti-self-dual Yang-Mills equation. Hence, we can define the following quantity and called it the action density in this paper:

$$
\operatorname{Tr} F^{2}:=\operatorname{Tr} F_{m n} F^{m n}=-2 \operatorname{Tr}\left(F_{w \widetilde{w}}^{2}+F_{z \widetilde{z}}^{2}+2 F_{\widetilde{z} w} F_{z \widetilde{w}}+2 F_{z w} F_{\widetilde{z} \widetilde{w}}\right)
$$

where $F^{m n}:=g^{m k} g^{n l} F_{k l}$. For ASD gauge fields, $\operatorname{Tr} F^{2}=4 \operatorname{Tr}\left(F_{w \widetilde{z}} F_{z \widetilde{w}}-F_{w \widetilde{w}}^{2}\right)$.

\footnotetext{
${ }^{1}$ Note that the relation between $J$ and $h, \widetilde{h}$ is different from $J:=\widetilde{h} h^{-1}$ in [11].
} 


\subsection{Soliton solutions and action densities for $G=\mathrm{GL}(2)$}

From now on, let us focus on soliton solutions for $G=\mathrm{GL}(2)$ generated from a trivial seed solution $J=1$ by the Darboux transformation [21].

The following $2 \times 2$ complex matrix $J$ is a solution of the Yang equation [21].

$$
J=-Q \Lambda^{-1} Q^{-1}
$$

where $\Lambda$ is a constant $2 \times 2$ matrix and $Q$ is a $2 \times 2$ matrix satisfying

$$
\partial_{w} Q=\left(\partial_{\widetilde{z}} Q\right) \Lambda, \partial_{z} Q=\left(\partial_{\widetilde{w}} Q\right) \Lambda
$$

Soliton solutions are given by setting $Q$ and $\Lambda$ as follows [11]:

$$
\begin{aligned}
Q=\left(\begin{array}{ll}
a_{1} e^{L}+a_{2} e^{-L} & b_{1} e^{M}+b_{2} e^{-M} \\
c_{1} e^{L}+c_{2} e^{-L} & d_{1} e^{M}+d_{2} e^{-M}
\end{array}\right), & \Lambda=\left(\begin{array}{ll}
\lambda & 0 \\
0 & \mu
\end{array}\right), \\
L:=\lambda \beta z+\alpha \widetilde{z}+\lambda \alpha w+\beta \widetilde{w}, & M:=\mu \delta z+\gamma \widetilde{z}+\mu \gamma w+\delta \widetilde{w},
\end{aligned}
$$

where $a_{1}, a_{2}, b_{1}, b_{2}, c_{1}, c_{2}, d_{1}, d_{2}, \alpha, \beta, \gamma, \delta, \lambda, \mu$ are complex constants. Note that we only consider this type of solution in this paper from now on, that is, $J$ and $Q$ in (2.8) and (2.10), respectively.

After a little bit lengthy calculation, we can obtain explicit form of the action density with respect to this solution (For the details, see appendix.):

$$
\operatorname{Tr} F^{2}=8(\lambda-\mu)^{2}(\alpha \delta-\beta \gamma)^{2} \varepsilon_{0} \widetilde{\varepsilon}_{0}\left[\frac{2 \varepsilon_{1} \widetilde{\varepsilon}_{1} \sinh ^{2} X_{1}-2 \varepsilon_{2} \widetilde{\varepsilon}_{2} \sinh ^{2} X_{2}-\varepsilon_{0} \widetilde{\varepsilon}_{0}}{\left(\left(\varepsilon_{1} \widetilde{\varepsilon}_{1}\right)^{\frac{1}{2}} \cosh X_{1}+\left(\varepsilon_{2} \widetilde{\varepsilon}_{2}\right)^{\frac{1}{2}} \cosh X_{2}\right)^{4}}\right]
$$

where

$$
\begin{aligned}
X_{1}:=M+L+\frac{1}{2} \log \left(\varepsilon_{1} / \widetilde{\varepsilon}_{1}\right), & X_{2}:=M-L+\frac{1}{2} \log \left(\varepsilon_{2} / \widetilde{\varepsilon}_{2}\right) \\
\varepsilon_{0}:=a_{2} c_{1}-a_{1} c_{2}, & \widetilde{\varepsilon_{0}}:=b_{2} d_{1}-b_{1} d_{2}, \\
\varepsilon_{1}:=a_{1} d_{1}-b_{1} c_{1}, & \widetilde{\varepsilon}_{1}:=a_{2} d_{2}-b_{2} c_{2}, \\
\varepsilon_{2}:=a_{2} d_{1}-b_{1} c_{2}, & \widetilde{\varepsilon}_{2}:=a_{1} d_{2}-b_{2} c_{1} .
\end{aligned}
$$

Note that the action density vanishes identically when $\lambda=\mu$ or $\alpha \delta=\beta \gamma$ or $\varepsilon_{0} \widetilde{\epsilon_{0}}=0$. Furthermore, $\varepsilon_{1} \widetilde{\epsilon_{1}}=\varepsilon_{2} \widetilde{\epsilon_{2}} \Longleftrightarrow \varepsilon_{0} \widetilde{\epsilon_{0}}=0$. This means the singularities appear on the locus $D:=\left\{(z, \widetilde{z}, w, \widetilde{w}) \in \mathbb{C}^{4} \mid\left(\varepsilon_{1} \widetilde{\varepsilon}_{1}\right)^{\frac{1}{2}} \cosh X_{1}+\left(\varepsilon_{2} \widetilde{\varepsilon}_{2}\right)^{\frac{1}{2}} \cosh X_{2}=0, \varepsilon_{1} \widetilde{\varepsilon}_{1} \neq \varepsilon_{2} \widetilde{\varepsilon}_{2}\right\}$ and $D$ is clearly nonempty because $X_{I}=i\left(n_{I}+1 / 2\right) \pi\left(I=1,2, n_{I} \in \mathbb{Z}\right)$ satisfies $\cosh X_{1}=$ $\cosh X_{2}=0$. To find other singularities, for example, we can impose a simple constraint: $\varepsilon_{1}=k \varepsilon_{2}, \widetilde{\varepsilon_{1}}=k \widetilde{\varepsilon_{2}}, k \in \mathbb{R} \backslash\{-1,0,1\}$ satisfying the condition $\varepsilon_{1} \widetilde{\varepsilon}_{1} \neq \varepsilon_{2} \widetilde{\varepsilon}_{2}$ so that phase shift factor $1 / 2 \log \left(\varepsilon_{1} / \widetilde{\varepsilon}_{1}\right)=1 / 2 \log \left(\varepsilon_{2} / \widetilde{\varepsilon}_{2}\right)=: \phi$. Then some classes of singularities would appear on the sub-locus of $\mathrm{D}$ :

$$
\widetilde{D}_{1}:=\left\{(z, \widetilde{z}, w, \widetilde{w}) \in \mathbb{C}^{4} \mid \tanh L \tanh (M+\phi)=-(|k|+1) /(|k|-1)\right\},
$$


and a special class of them can be found explicitly on the intersection of complex hyperplanes defined by $L=i\left\{\arctan ((|k|+1) /(|k|-1))+n_{L} \pi\right\} \quad$ and $\quad M=i\left(n_{M}+1 / 4\right) \pi-$ $\phi\left(n_{L}, n_{M} \in \mathbb{Z}\right)$. Next let us consider another example of sub-locus of $D$ :

$$
\widetilde{D}_{2}:=D \cap\left\{(z, \widetilde{z}, w, \widetilde{w}) \in \mathbb{C}^{4} \mid \operatorname{Im} X_{I}=n_{I} \pi, n_{I} \in \mathbb{Z}, I=1,2\right\},
$$

which would greatly simplify the problem of locus $D$ to a homogeneous system of two linear equations of $\cosh \left(\operatorname{Re} X_{I}\right)$ by the argument formula: $\cosh X_{I}=\cosh \left(\operatorname{Re} X_{I}+i n_{I} \pi\right)=$ $(-1)^{n_{I}} \cosh \left(\operatorname{Re} X_{I}\right)$. Then under the condition: $\Delta:=\left|\begin{array}{l}\operatorname{Re}\left(\varepsilon_{1} \widetilde{\varepsilon}_{1}\right)^{1 / 2} \operatorname{Re}\left(\varepsilon_{2} \widetilde{\varepsilon}_{2}\right)^{1 / 2} \\ \operatorname{Im}\left(\varepsilon_{1} \widetilde{\varepsilon}_{1}\right)^{1 / 2} \operatorname{Im}\left(\varepsilon_{2} \widetilde{\varepsilon}_{2}\right)^{1 / 2}\end{array}\right| \neq 0$, the singularities of action density are removed successfully since the singular sub-locus $\widetilde{D}_{2}$ becomes empty.

Classifying all the singularities of the complex action density (2.12) in details is a rewarding job, however, we would like to discuss this issue in a separated paper because our aim in this paper is to study the real-valued action density for physical purpose. In fact, we can exclude all the singularities on each real slice by adjusting parameters $a_{I}, b_{I}, c_{I}, d_{I}$ in (2.10) and taking reality conditions on (2.11). The remaining details are discussed in section 3 .

On the other hand, we also find that the action density has two principal peaks lie on $\operatorname{Re} X_{1}=0$ and $\operatorname{Re} X_{2}=0$ on the no singularity region, as we mentioned in (2.18). This fact is very interesting and quite different from our experience in the lower-dimensional soliton equations. More precisely, the principal peaks of soliton configurations of lowerdimensional soliton equations usually lie on the $\operatorname{Re} L=0$ or $\operatorname{Re} M=0$ (up to phase shift factors), however, our principal peaks lie on $\operatorname{Re}(M \pm L)=0$. Let us consider the same analysis of the anti-self-dual Yang-Mills equation, like that for lower-dimensional solition equations. Firstly, we take a limit of $r^{2}:=\left|\sum_{m=1}^{4} z^{m} \bar{z}^{m}\right|^{2} \rightarrow \infty$ so that $|L|$ is finite in the solution (2.10). Then $\left|e^{M}\right|$ goes to infinity or zero and $\left|e^{-M}\right|$ goes to zero or infinity, respectively. That is,

$$
Q \stackrel{r^{2} \rightarrow \infty}{\longrightarrow}\left(\begin{array}{cc}
a_{1} e^{L}+a_{2} e^{-L} & b_{1} e^{M} \\
c_{1} e^{L}+c_{2} e^{-L} & d_{1} e^{M}
\end{array}\right) \text { or }\left(\begin{array}{cc}
a_{1} e^{L}+a_{2} e^{-L} & b_{2} e^{-M} \\
c_{1} e^{L}+c_{2} e^{-L} & d_{2} e^{-M}
\end{array}\right) .
$$

Note that the former and latter cases correspond to $\left(b_{2}, d_{2}\right)=(0,0)$ and $\left(b_{1}, d_{1}\right)=(0,0)$, respectively. By comparing (2.12), the resulting action density vanishes in the both cases while in the lower-dimensional soliton equations, the configuration has its principal peak on $\operatorname{Re} L=0$ by similar analysis.

Inspired from the above analysis, let us focus on one principal peak of (2.12) and set $a_{2}=b_{1}=c_{1}=d_{2}=0$. Then we can obtain a reduced form of our soliton solution

$$
Q=\left(\begin{array}{cc}
a e^{L} & b e^{-M} \\
c e^{-L} & d e^{M}
\end{array}\right), \quad a, b, c, d \in \mathbb{C},
$$

which leads to a simpler form of action density:

$$
\operatorname{Tr} F^{2}=8(\lambda-\mu)^{2}(\alpha \delta-\beta \gamma)^{2}\left(2 \operatorname{sech}^{2} X-3 \operatorname{sech}^{4} X\right),
$$

where $X:=M+L+\frac{1}{2} \log (-a d / b c)$. (Note that $\varepsilon_{0} \widetilde{\varepsilon}_{0}=\varepsilon_{1} \widetilde{\varepsilon}_{1}=-a b c d, \varepsilon_{2} \widetilde{\varepsilon}_{2}=0$.) 
Now let us discuss the singularity problem of the reduced action density (2.21) by the following argument formula of hyperbolic functions:

$$
\operatorname{sech}^{2}(x+i y)=2\left[\frac{\cosh 2 x \cos 2 y+1}{(\cosh 2 x+\cos 2 y)^{2}}-i \frac{\sinh 2 x \sin 2 y}{(\cosh 2 x+\cos 2 y)^{2}}\right] .
$$

We find that (2.21) has periodicity on the slice spaces: $X=a+i \operatorname{Im} X$ for any given real number $a$, and the singularities appear periodically in the case of $a=0$ because $\operatorname{sech}^{2} X=\sec ^{2}(\operatorname{Im} X)$ if $X=i \operatorname{Im} X$. Therefore, (2.21) has no solitonic behavior on slice spaces if the real part of $\mathrm{X}$ is fixed. On the other hand, to remove the singularities and periodicity, we can impose some constraint on the imaginary part of $X$. For example, taking the condition $X=\operatorname{Re} X+i n \pi$ for $n \in \mathbb{Z}$ would achieve this goal as the following:

$$
\operatorname{Tr} F^{2}=8(\lambda-\mu)^{2}(\alpha \delta-\beta \gamma)^{2}\left(2 \operatorname{sech}^{2}(\operatorname{Re} X)-3 \operatorname{sech}^{4}(\operatorname{Re} X)\right),
$$

which possesses real-valued solitonic behavior up to complex constants. For other nontrivial examples, we can use formula (2.22) and consider the slice spaces: $X=\operatorname{Re} X+i(n \pm 1 / 4) \pi$ for $n \in \mathbb{Z}$ to get the result:

$$
\begin{aligned}
2 \operatorname{sech}^{2} X-3 \operatorname{sech}^{4} X= & 8\left(2 \operatorname{sech}^{2}(2 \operatorname{Re} X)-3 \operatorname{sech}^{4}(2 \operatorname{Re} X)\right) \\
& \pm 4 i(6 \operatorname{sech}(2 \operatorname{Re} X)-1) \operatorname{sech}^{2}(2 \operatorname{Re} X) \tanh (2 \operatorname{Re} X) .
\end{aligned}
$$

Note that (2.24) which belongs to a new class of solutions, is quite different from (2.23) because the solitonic behavior appears in both real part and imaginary part.

Finally, we remark a condition for $J$-matrix such that $J$ is unitary. We hope that our understanding of $J$-matrix would be helpful for the realization of $G=\mathrm{U}(N)$ since the action density becomes real-valued and fit to physical interpretation when $G=\mathrm{U}(N)$. Let us put a condition on the solution $Q$ in (2.8) as follows

$$
Q=\left(\begin{array}{cc}
A & B \\
-\bar{B} & \bar{A}
\end{array}\right), \quad \Lambda=\left(\begin{array}{cc}
\lambda & 0 \\
0 & \mu
\end{array}\right)
$$

Then Yang's $J$ matrix becomes

$$
J=\frac{-1}{|A|^{2}+|B|^{2}}\left(\begin{array}{cc}
(1 / \lambda)|A|^{2}+(1 / \mu)|B|^{2} & (1 / \mu-1 / \lambda) A B \\
(1 / \mu-1 / \lambda) \overline{A B} & (1 / \mu)|A|^{2}+(1 / \lambda)|B|^{2}
\end{array}\right) .
$$

Hence, we can find that under the condition (2.25), $J \in \mathrm{U}(2) \Leftrightarrow|\lambda|=|\mu|=1$ and $J \in \mathrm{SU}(2) \Leftrightarrow \mu=\bar{\lambda}$ and $|\lambda|=1$.

We will see it soon in the next section that the ansatz (2.25) gives a magical way to construct $\operatorname{Im} X=0$ type action densities (3.10), (3.22), (3.33), and (3.44) belonging to the same class $(n=0)$ of $(2.23)$.

\section{Soliton solutions on four-dimensional real spaces}

In this section, we construct soliton solutions on four-dimensional real spaces with three kinds of signatures and the corresponding action densities could be realized to real-valued 
functions by taking the reality conditions in section 2.1 and condition (2.25). More precisely, $c_{1}=-\bar{b}_{1}, c_{2}=-\bar{b}_{2}, d_{1}=\bar{a}_{1}, d_{2}=\bar{a}_{2}$ and $\bar{M}=L$. The latter condition gives rise to relations between parameters $\alpha, \beta, \gamma, \delta, \lambda, \mu$ on each real slice. After these replacements, the action density $\operatorname{Tr} F^{2}$ reduces to the standard one: $\operatorname{Tr} F_{\mu \nu} F^{\mu \nu}$ with respect to local coordinates $x^{\mu}(\mu=0,1,2,3)$ on the four-dimensional real spaces. We can even show that the action density $\operatorname{Tr} F_{\mu \nu} F^{\mu \nu}$ is real-valued because $X_{1}$ becomes real and $X_{2}$ becomes pure imaginary.

More interestingly, the soliton solutions (2.20) represent domain wall solutions and the integration of the corresponding action densities over the real spaces are suggested to be infinity but zero. We put the proof in section 3.1. This property might shed light on a new study area of domain walls in cosmology.

On the other hand, we will see that $G=\mathrm{U}(N)$ can be realized only on the Ultrahyperbolic space $\mathbb{U}_{2}$ in section 3.4 because both gauge fields $A_{\mu}$ and field strengths $F_{\mu \nu}$ must take values in anti-hermitian $N \times N$ matrices when $G=\mathrm{U}(N)$.

\subsection{On Euclidean real space $\mathbb{E}$}

To realize the Euclidean real slice condition: $\widetilde{z}=\bar{z}, \widetilde{w}=-\bar{w}$, we take the following combination of the real coordinates $x^{0}, x^{1}, x^{2}, x^{3}$ on $\mathbb{E}$ :

$$
z=\frac{1}{\sqrt{2}}\left(x^{0}-i x^{1}\right), \widetilde{z}=\frac{1}{\sqrt{2}}\left(x^{0}+i x^{1}\right), w=-\frac{1}{\sqrt{2}}\left(x^{2}-i x^{3}\right), \widetilde{w}=\frac{1}{\sqrt{2}}\left(x^{2}+i x^{3}\right),
$$

which satisfy the Euclidean metric $d s^{2}=\left(d x^{0}\right)^{2}+\left(d x^{1}\right)^{2}+\left(d x^{2}\right)^{2}+\left(d x^{3}\right)^{2}$. Then eq. (2.3) reduces to the anti-self-dual Yang-Mills equation: $F_{01}+F_{23}=0, \quad F_{02}-F_{13}=0, F_{03}+F_{12}=0$.

Further, the condition $\bar{M}=L$ gives rise to the relations $\gamma=\overline{\lambda \beta}, \delta=-\bar{\lambda} \bar{\alpha}, \mu=-1 / \bar{\lambda}$, and the soliton solution (2.10) could be represented by

$$
Q=\left(\begin{array}{cc}
a_{1} e^{L}+a_{2} e^{-L} & b_{1} e^{\bar{L}}+b_{2} e^{-\bar{L}} \\
-\bar{b}_{1} e^{L}-\bar{b}_{2} e^{-L} & \bar{a}_{1} e^{\bar{L}}+\bar{a}_{2} e^{-\bar{L}}
\end{array}\right), \quad \Lambda=\left(\begin{array}{cc}
\lambda & 0 \\
0 & -1 / \bar{\lambda}
\end{array}\right),
$$

where $L=(\lambda \beta) z+\alpha \bar{z}+(\lambda \alpha) w-\beta \bar{w}$. The real coordinates expansion of it is

$$
L=l_{\mu} x^{\mu}, \quad l_{\mu}=\frac{1}{\sqrt{2}}(\alpha+\lambda \beta, i(\alpha-\lambda \beta), \beta-\lambda \alpha, i(\beta+\lambda \alpha)) .
$$

Under these setting, the action density of the soliton solution (3.2) is

$$
\operatorname{Tr} F_{\mu \nu} F^{\mu \nu}=8\left[\left(|\alpha|^{2}+|\beta|^{2}\right)\left(|\lambda|^{2}+1\right)\left|\varepsilon_{0}\right|\right]^{2}\left[\frac{2 \varepsilon_{1} \widetilde{\varepsilon}_{1} \sinh ^{2} X_{1}-2\left|\varepsilon_{2}\right|^{2} \sinh ^{2} X_{2}-\left|\varepsilon_{0}\right|^{2}}{\left(\left(\varepsilon_{1} \widetilde{\varepsilon}_{1}\right)^{\frac{1}{2}} \cosh X_{1}+\left|\varepsilon_{2}\right| \cosh X_{2}\right)^{4}}\right],
$$

where

$$
\begin{aligned}
X_{1} & =\bar{L}+L+\frac{1}{2} \log \left(\varepsilon_{1} / \widetilde{\varepsilon}_{1}\right), & & X_{2}=\bar{L}-L+\frac{1}{2} \log \left(\varepsilon_{2} / \bar{\varepsilon}_{2}\right), \\
\varepsilon_{0} & =a_{1} \bar{b}_{2}-a_{2} \bar{b}_{1}, & & \widetilde{\varepsilon}_{1}=\left|a_{2}\right|^{2}+\left|b_{2}\right|^{2} \in \mathbb{R}, \\
\varepsilon_{1} & =\left|a_{1}\right|^{2}+\left|b_{1}\right|^{2}, & & \\
\varepsilon_{2} & =\bar{a}_{1} a_{2}+b_{1} \bar{b}_{2} . & &
\end{aligned}
$$

Note that the action density vanishes identically when $\alpha=\beta=0$ or $\varepsilon_{0}=0$. 
To realize the gauge group to be $G=\mathrm{U}(N)$, the action density $\operatorname{Tr} F_{\mu \nu} F^{\mu \nu}$ should be negative definite because $F_{\mu \nu}$ is anti-hermitian and eigenvalues of it are pure imaginary. However, the action density (3.4) is not negative definite at any point on $\mathbb{E}$. This implies that the gauge group cannot be unitary.

However, action density $\operatorname{Tr} F_{\mu \nu} F^{\mu \nu}$ could be real-valued even though gauge group is not unitary. Note that this configuration has solitonic behavior in the $X_{1}$-direction and periodic behavior in the $X_{2}$-direction because $X_{1}$ is clearly real and $X_{2}$ is pureimaginary, implying $\cosh X_{2}=\cos \left(\operatorname{Im} X_{2}\right), \sinh X_{2}=i \sin \left(\operatorname{Im} X_{2}\right)$. By this property and (3.5) (3.8), $\operatorname{Tr} F_{\mu \nu} F^{\mu \nu}$ is clearly real-valued. Furthermore, since $\cosh X_{1} \geq 1,-1 \leq$ $\cosh X_{2}=\cos \left(\operatorname{Im} X_{2}\right) \leq 1$ and $\varepsilon_{1} \widetilde{\varepsilon}_{1} \geq\left|\varepsilon_{2}\right|^{2}$, all singularities appear on the locus $D:=$ $\left\{\left.x^{\mu} \in \mathbb{R}^{4}\left|\cosh X_{1}=1, \cosh X_{2}=\cos \left(\operatorname{Im} X_{2}\right)=-1, \varepsilon_{1} \widetilde{\varepsilon}_{1}=\right| \varepsilon_{2}\right|^{2}\right\}$. As we mentioned in section 2.2 below (2.16), the final condition $\varepsilon_{1} \widetilde{\varepsilon}_{1}=\left|\varepsilon_{2}\right|^{2}$ in $D$ implies $\operatorname{Tr} F_{\mu \nu} F^{\mu \nu}=0$. Therefore, there is no singularity in the action density. The same discussion is also valid for other signatures.

Another surprising thing comes when we focus only on solitonic behavior part by setting $a_{2}=b_{1}=0$ in (3.4). Then the soliton solution

$$
Q=\left(\begin{array}{cc}
a e^{L} & b e^{-\bar{L}} \\
-\bar{b} e^{-L} & \bar{a} e^{\bar{L}}
\end{array}\right),
$$

leads to a simpler form of action density:

$$
\operatorname{Tr} F_{\mu \nu} F^{\mu \nu}=8\left[\left(|\alpha|^{2}+|\beta|^{2}\right)\left(|\lambda|^{2}+1\right)\right]^{2}\left(2 \operatorname{sech}^{2} X-3 \operatorname{sech}^{4} X\right),
$$

where $X=L+\bar{L}+\log (|a| /|b|)$. (Note that $\left|\varepsilon_{0}\right|^{2}=\varepsilon_{1} \widetilde{\varepsilon}_{1}=|a b|^{2}, \quad\left|\varepsilon_{2}\right|^{2}=0$.)

We find that the action density has its principal peak on a three-dimensional hyperplane defined by $X=L+\bar{L}+\log (|a| /|b|)=0$ with normal vector $l_{\mu}+\bar{l}_{\mu}$. Therefore, it's a domain wall in $\mathbb{R}^{4}$. More surprisingly, integration of this action density over $\mathbb{E}$ is suggested to be zero. In order to explain this property, let us introduce three independent axes $X^{1}, X^{2}, X^{3}$ in the directions orthogonal to the $X$-axis (normal direction of the domain wall $(\mathrm{DW}))$. Then, integration of the action density would be performed naively by the following finite box regularization:

$$
\begin{aligned}
\int_{\mathbb{E}} \operatorname{Tr} F_{\mu \nu} F^{\mu \nu} d^{4} x & \propto \lim _{R \rightarrow \infty} \int_{-R}^{R} \int_{-R}^{R} \int_{-R}^{R} d X^{1} d X^{2} d X^{3} \int_{-R}^{R}\left(2 \operatorname{sech}^{2} X-3 \operatorname{sech}^{4} X\right) d X \\
& =\int_{\text {DW }} d X^{1} d X^{2} d X^{3} \int_{-\infty}^{\infty}\left(2 \operatorname{sech}^{2} X-3 \operatorname{sech}^{4} X\right) d X \\
& =\left.\int_{\text {DW }} d X^{1} d X^{2} d X^{3}\left(\tanh X \cdot \operatorname{sech}^{2} X\right)\right|_{-\infty} ^{\infty}=0
\end{aligned}
$$

This result suggests that the soliton solution (3.9) belongs to the sector of instanton number zero. The same discussion is also valid for other signatures. We note that the present discussion lacks mathematical rigor. In order to justify the integration, we have to solve the anti-self-dual Yang-Mills equation originally with a suitable boundary condition compatible to the box regularization. ${ }^{2}$ This issue will be reported elsewhere.

\footnotetext{
${ }^{2}$ The authors thank an anonymous referee to point this out.
} 
Finally, we remark that $J \in \mathrm{U}(2) \Leftrightarrow \Lambda=\left(\begin{array}{cc}e^{i \theta} & 0 \\ 0 & -e^{i \theta}\end{array}\right)$ and $J \in \mathrm{SU}(2) \Leftrightarrow \Lambda=$ $\pm\left(\begin{array}{cc}i & 0 \\ 0 & -i\end{array}\right)(\theta \in \mathbb{R})$ on $\mathbb{E}$

In this section, we call solutions like (3.2) one-soliton solutions and solutions like (3.9) pure one-soliton solutions to distinguish them.

\subsection{On Minkowski Real Space $\mathbb{M}$}

As discussed in the Euclidean case, we can take the following combination of real coordinates $x^{0}, x^{1}, x^{2}, x^{3}$ on $\mathbb{M}$ to realize the real slice condition: $z, \widetilde{z} \in \mathbb{R}, \widetilde{w}=\bar{w}$

$$
z=\frac{1}{\sqrt{2}}\left(x^{0}-x^{1}\right), \widetilde{z}=\frac{1}{\sqrt{2}}\left(x^{0}+x^{1}\right), w=\frac{1}{\sqrt{2}}\left(x^{2}-i x^{3}\right), \widetilde{w}=\frac{1}{\sqrt{2}}\left(x^{2}+i x^{3}\right),
$$

which satisfy the Minkowski metric $d s^{2}=\left(d x^{0}\right)^{2}-\left(d x^{1}\right)^{2}-\left(d x^{2}\right)^{2}-\left(d x^{3}\right)^{2}$. Then eq. (2.3) reduces to the anti-self-dual Yang-Mills equation: $F_{01}+i F_{23}=0, \quad F_{02}-i F_{13}=0, \quad F_{03}+$ $i F_{12}=0$. Due to the ASD equation, the realization of gauge group $G=\mathrm{U}(N)$ is impossible since gauge fields $A_{0}, A_{1}, A_{2}$ and $A_{3}$ could not be all anti-hermitian.

Further, the condition $\bar{M}=L$ yields relations $\beta=\bar{\mu} \alpha, \gamma=\bar{\alpha}, \delta=\bar{\lambda} \bar{\alpha}$ (Relation between $\lambda$ and $\mu$ is not necessary.) and the one-soliton solution (2.10) could be represented by

$$
\begin{aligned}
Q & =\left(\begin{array}{cc}
a_{1} e^{L}+a_{2} e^{-L} & b_{1} e^{\bar{L}}+b_{2} e^{-\bar{L}} \\
-\bar{b}_{1} e^{L}-\bar{b}_{2}^{-L} & \bar{a}_{1} e^{\bar{L}}+\bar{a}_{2} e^{-\bar{L}}
\end{array}\right), \quad \Lambda=\left(\begin{array}{cc}
\lambda & 0 \\
0 & \mu
\end{array}\right), \\
L & =(\lambda \bar{\mu} \alpha) z+\alpha \widetilde{z}+(\lambda \alpha) w+(\bar{\mu} \alpha) \bar{w} \\
& =l_{\mu} x^{\mu}, \quad l_{\mu}=\frac{1}{\sqrt{2}}((1+\lambda \bar{\mu}) \alpha,(1-\lambda \bar{\mu}) \alpha,(\bar{\mu}+\lambda) \alpha, i(\bar{\mu}-\lambda) \alpha) .
\end{aligned}
$$

Under these setting, the action density of the solution (3.13) is

$$
\operatorname{Tr} F_{\mu \nu} F^{\mu \nu}=8|\alpha(\lambda-\mu)|^{4}\left|\varepsilon_{0}\right|^{2}\left[\frac{2 \varepsilon_{1} \widetilde{\varepsilon}_{1} \sinh ^{2} X_{1}-2\left|\varepsilon_{2}\right|^{2} \sinh ^{2} X_{2}-\left|\varepsilon_{0}\right|^{2}}{\left(\left(\varepsilon_{1} \widetilde{\varepsilon}_{1}\right)^{\frac{1}{2}} \cosh X_{1}+\left|\varepsilon_{2}\right| \cosh X_{2}\right)^{4}}\right],
$$

where

$$
\begin{aligned}
X_{1} & =\bar{L}+L+\frac{1}{2} \log \left(\varepsilon_{1} / \widetilde{\varepsilon}_{1}\right), & & X_{2}=\bar{L}-L+\frac{1}{2} \log \left(\varepsilon_{2} / \bar{\varepsilon}_{2}\right), \\
\varepsilon_{0} & =a_{1} \bar{b}_{2}-a_{2} \bar{b}_{1}, & & \widetilde{\varepsilon}_{1}=\left|a_{2}\right|^{2}+\left|b_{2}\right|^{2} \in \mathbb{R}, \\
\varepsilon_{1} & =\left|a_{1}\right|^{2}+\left|b_{1}\right|^{2}, & & \\
\varepsilon_{2} & =\bar{a}_{1} a_{2}+b_{1} \bar{b}_{2} . & &
\end{aligned}
$$

Note that the action density vanishes identically when $\lambda=\mu$ or $\alpha=0$ or $\varepsilon_{0}=0$.

The pure one-soliton solution is given by the same trick as in the Euclidean case:

$$
Q=\left(\begin{array}{cc}
a e^{L} & b e^{-\bar{L}} \\
-\bar{b} e^{-L} & \bar{a} e^{\bar{L}}
\end{array}\right)
$$


which leads to the action density

$$
\operatorname{Tr} F_{\mu \nu} F^{\mu \nu}=8\left|\alpha^{2}(\lambda-\mu)\right|^{2}\left(2 \operatorname{sech}^{2} X-3 \operatorname{sech}^{4} X\right),
$$

where $X=L+\bar{L}+\log (|a| /|b|)$. (Note that $\left|\varepsilon_{0}\right|^{2}=\varepsilon_{1} \widetilde{\varepsilon}_{1}=|a b|^{2},\left|\varepsilon_{2}\right|^{2}=0$.) Once again, integration of the action density (3.22) over $\mathbb{M}$ vanishes by the same reason as in (3.11).

Finally, we remark that $J \in \mathrm{U}(2) \Leftrightarrow \Lambda=\left(\begin{array}{cc}e^{i \theta_{1}} & 0 \\ 0 & e^{i \theta_{2}}\end{array}\right)$ and $J \in \mathrm{SU}(2) \Leftrightarrow \Lambda=$ $\left(\begin{array}{cc}e^{i \theta} & 0 \\ 0 & e^{-i \theta}\end{array}\right)\left(\theta_{1}, \theta_{2} \in \mathbb{R}\right)$ on $\mathbb{M}$.

\subsection{On ultrahyperbolic real space $\mathbb{U}_{1}$}

The discussion of $\mathbb{U}_{1}$ is quite similar to the Euclidean case. We can take the following combination of the real coordinates $x^{0}, x^{1}, x^{2}, x^{3}$ on $\mathbb{U}_{1}$ to realize the real slice condition: $\widetilde{z}=\bar{z}, \widetilde{w}=\bar{w}$

$$
z=\frac{1}{\sqrt{2}}\left(x^{0}-i x^{1}\right), \widetilde{z}=\frac{1}{\sqrt{2}}\left(x^{0}+i x^{1}\right), w=\frac{1}{\sqrt{2}}\left(x^{2}-i x^{3}\right), \widetilde{w}=\frac{1}{\sqrt{2}}\left(x^{2}+i x^{3}\right),
$$

which satisfy the Ultrahyperbolic metric $d s^{2}=\left(d x^{0}\right)^{2}+\left(d x^{1}\right)^{2}-\left(d x^{2}\right)^{2}-\left(d x^{3}\right)^{2}$. Then eq. (2.3) reduces to the anti-self-dual Yang-Mills equation: $F_{01}-F_{23}=0, \quad F_{02}-F_{13}=0$, $F_{03}+F_{12}=0$.

Further, the condition $\bar{M}=L$ yields the relations $\gamma=\overline{\lambda \beta}, \delta=\bar{\lambda} \bar{\alpha}, \mu=1 / \bar{\lambda}$, and the one-soliton solution (2.10) could be represented by

$$
\begin{aligned}
Q & =\left(\begin{array}{cc}
a_{1} e^{L}+a_{2} e^{-L} & b_{1} e^{\bar{L}}+b_{2} e^{-\bar{L}} \\
-\bar{b}_{1} e^{L}-\bar{b}_{2} e^{-L} & \bar{a}_{1} e^{\bar{L}}+\bar{a}_{2} e^{-\bar{L}}
\end{array}\right), \quad \Lambda=\left(\begin{array}{cc}
\lambda & 0 \\
0 & 1 / \bar{\lambda}
\end{array}\right), \\
L & =(\lambda \beta) z+\alpha \bar{z}+(\lambda \alpha) w+\beta \bar{w} \\
& =l_{\mu} x^{\mu}, \quad l_{\mu}=\frac{1}{\sqrt{2}}(\alpha+\lambda \beta, i(\alpha-\lambda \beta), \beta+\lambda \alpha, i(\beta-\lambda \alpha)) .
\end{aligned}
$$

Under these setting, the action density of the solution (3.24) is

$$
\operatorname{Tr} F_{\mu \nu} F^{\mu \nu}=8\left[\left(|\alpha|^{2}-|\beta|^{2}\right)\left(|\lambda|^{2}-1\right)\left|\varepsilon_{0}\right|\right]^{2}\left[\frac{2 \varepsilon_{1} \widetilde{\varepsilon}_{1} \sinh ^{2} X_{1}-2\left|\varepsilon_{2}\right|^{2} \sinh ^{2} X_{2}-\left|\varepsilon_{0}\right|^{2}}{\left(\left(\varepsilon_{1} \widetilde{\varepsilon}_{1}\right)^{\frac{1}{2}} \cosh X_{1}+\left|\varepsilon_{2}\right| \cosh X_{2}\right)^{4}}\right],
$$

where

$$
\begin{array}{rlrl}
X_{1} & =\bar{L}+L+\frac{1}{2} \log \left(\varepsilon_{1} / \widetilde{\varepsilon}_{1}\right), & & X_{2}=\bar{L}-L+\frac{1}{2} \log \left(\varepsilon_{2} / \bar{\varepsilon}_{2}\right), \\
\varepsilon_{0} & =a_{1} \bar{b}_{2}-a_{2} \bar{b}_{1}, & & \\
\varepsilon_{1} & =\left|a_{1}\right|^{2}+\left|b_{1}\right|^{2}, & \widetilde{\varepsilon}_{1}=\left|a_{2}\right|^{2}+\left|b_{2}\right|^{2} \in \mathbb{R}, \\
\varepsilon_{2} & =\bar{a}_{1} a_{2}+b_{1} \bar{b}_{2} . & &
\end{array}
$$

Note that the action density vanishes identically when $|\alpha|=|\beta|$ or $|\lambda|=1$ or $\varepsilon_{0}=0$. 
The pure one-soliton solution is given by

$$
Q=\left(\begin{array}{cc}
a e^{L} & b e^{-\bar{L}} \\
-\bar{b} e^{-L} & \bar{a} e^{\bar{L}}
\end{array}\right)
$$

which leads to the action density

$$
\operatorname{Tr} F_{\mu \nu} F^{\mu \nu}=8\left[\left(|\alpha|^{2}-|\beta|^{2}\right)\left(|\lambda|^{2}-1\right)\right]^{2}\left(2 \operatorname{sech}^{2} X-3 \operatorname{sech}^{4} X\right),
$$

where $X=L+\bar{L}+\log (|a| /|b|)$. (Note that $\left|\varepsilon_{0}\right|^{2}=\varepsilon_{1} \widetilde{\varepsilon}_{1}=|a b|^{2}, \quad\left|\varepsilon_{2}\right|^{2}=0$.) Integration of the action density (3.33) over $\mathbb{U}_{1}$ is zero again by the same reason as in (3.11).

Finally, we remark that the condition $J \in \mathrm{U}(2)$ implies $\operatorname{Tr} F_{\mu \nu} F^{\mu \nu}=0$ on $\mathbb{U}_{1}$. In fact, the gauge group can not be unitary under the gauge condition $A_{\widetilde{z}}=A_{\widetilde{w}}=0$ on $\mathbb{U}_{1}$ as well because $\sqrt{2} A_{\widetilde{z}}=A_{0}-i A_{1}, \sqrt{2} A_{\widetilde{w}}=A_{2}-i A_{3}$ implies $A_{0}=i A_{1}, A_{2}=i A_{3}$. Hence under this gauge, only one possible solution is $A_{\mu}=0$ for $G=\mathrm{U}(N)$, that is, $F_{\mu \nu}=0$. The vanishing field strength leads to the trivial action density $\operatorname{Tr} F_{\mu \nu} F^{\mu \nu}=0$ which is valid in arbitrary gauge. Therefore there is no $G=\mathrm{U}(N)$ ASD gauge fields which give non-trivial action density.

\subsection{On ultrahyperbolic real space $\mathbb{U}_{2}$}

Finally, we discuss another real slice of the Ultrahyperbolic signature, say $\mathbb{U}_{2}$. We take the following combination of real coordinates $x^{0}, x^{1}, x^{2}, x^{3}$ on $\mathbb{U}_{2}$ to realize the real slice condition: $z, \widetilde{z}, w, \widetilde{w} \in \mathbb{R}$

$$
z=\frac{1}{\sqrt{2}}\left(x^{0}-x^{2}\right), \widetilde{z}=\frac{1}{\sqrt{2}}\left(x^{0}+x^{2}\right), w=-\frac{1}{\sqrt{2}}\left(x^{1}-x^{3}\right), \widetilde{w}=\frac{1}{\sqrt{2}}\left(x^{1}+x^{3}\right),
$$

which satisfy the Ultrahyperbolic signature $d s^{2}=\left(d x^{0}\right)^{2}+\left(d x^{1}\right)^{2}-\left(d x^{2}\right)^{2}-\left(d x^{3}\right)^{2}$. Then eq. (2.3) reduces to the anti-self-dual Yang-Mills equation: $F_{01}+F_{23}=0, \quad F_{02}+F_{13}=$ $0, \quad F_{03}-F_{12}=0$.

Further, the one-soliton solution (2.10) is reduced by the condition $\bar{M}=L(\Rightarrow \gamma=$ $\bar{\alpha}, \delta=\bar{\beta}, \mu=\bar{\lambda})$ to the following:

$$
\begin{aligned}
Q & =\left(\begin{array}{cc}
a_{1} e^{L}+a_{2} e^{-L} & b_{1} e^{\bar{L}}+b_{2} e^{-\bar{L}} \\
-\bar{b}_{1} e^{L}-\bar{b}_{2} e^{-L} & \bar{a}_{1} e^{\bar{L}}+\bar{a}_{2} e^{-\bar{L}}
\end{array}\right), \quad \Lambda=\left(\begin{array}{cc}
\lambda & 0 \\
0 & \bar{\lambda}
\end{array}\right), \\
L & =(\lambda \beta) z+\alpha \widetilde{z}+(\lambda \alpha) w+\beta \widetilde{w}, \\
& =l_{\mu} x^{\mu}, \quad l_{\mu}=\frac{1}{\sqrt{2}}(\alpha+\lambda \beta, \beta-\lambda \alpha, \alpha-\lambda \beta, \beta+\lambda \alpha),
\end{aligned}
$$

which leads to the action density

$$
\operatorname{Tr} F_{\mu \nu} F^{\mu \nu}=8\left[(\alpha \bar{\beta}-\bar{\alpha} \beta)(\lambda-\bar{\lambda})\left|\varepsilon_{0}\right|\right]^{2}\left[\frac{2 \varepsilon_{1} \widetilde{\varepsilon}_{1} \sinh ^{2} X_{1}-2\left|\varepsilon_{2}\right|^{2} \sinh ^{2} X_{2}-\left|\varepsilon_{0}\right|^{2}}{\left(\left(\varepsilon_{1} \widetilde{\varepsilon}_{1}\right)^{\frac{1}{2}} \cosh X_{1}+\left|\varepsilon_{2}\right| \cosh X_{2}\right)^{4}}\right],
$$


where

$$
\begin{array}{rlrl}
X_{1} & =\bar{L}+L+\frac{1}{2} \log \left(\varepsilon_{1} / \widetilde{\varepsilon}_{1}\right), & & X_{2}=\bar{L}-L+\frac{1}{2} \log \left(\varepsilon_{2} / \bar{\varepsilon}_{2}\right), \\
\varepsilon_{0} & =a_{1} \bar{b}_{2}-a_{2} \bar{b}_{1}, & & \\
\varepsilon_{1} & =\left|a_{1}\right|^{2}+\left|b_{1}\right|^{2}, & \widetilde{\varepsilon}_{1}=\left|a_{2}\right|^{2}+\left|b_{2}\right|^{2} \in \mathbb{R}, \\
\varepsilon_{2} & =\bar{a}_{1} a_{2}+b_{1} \bar{b}_{2} . & &
\end{array}
$$

Note that the action density vanishes identically when $\alpha \bar{\beta} \in \mathbb{R}$ or $\lambda \in \mathbb{R}$ or $\varepsilon_{0}=0$.

The pure one-soliton solution is given by

$$
Q=\left(\begin{array}{cc}
a e^{L} & b e^{-\bar{L}} \\
-\bar{b} e^{-L} & \bar{a} e^{\bar{L}}
\end{array}\right),
$$

and the action density becomes

$$
\operatorname{Tr} F_{\mu \nu} F^{\mu \nu}=8[(\alpha \bar{\beta}-\bar{\alpha} \beta)(\lambda-\bar{\lambda})]^{2}\left(2 \operatorname{sech}^{2} X-3 \operatorname{sech}^{4} X\right),
$$

where $X=L+\bar{L}+\log (|a| /|b|)$. (Note that $\left|\varepsilon_{0}\right|^{2}=\varepsilon_{1} \widetilde{\varepsilon}_{1}=|a b|^{2}, \quad\left|\varepsilon_{2}\right|^{2}=0$.) By the same reason as in (3.11), integration of the action density (3.44) over $\mathbb{U}_{2}$ is zero.

Finally, we remark that $J \in \mathrm{U}(2) \Leftrightarrow J \in \mathrm{SU}(2) \Leftrightarrow \Lambda=\left(\begin{array}{cc}e^{i \theta} & 0 \\ 0 & e^{-i \theta}\end{array}\right)$ on $\mathbb{U}_{2}$. In fact, we even find that the gauge group can be unitary in this case! First of all, gauge fields $A_{z}$ and $A_{w}$ are anti-hermitian on $\mathbb{U}_{2}$ naturally (See (A.5), (A.6)). On the other hand, $\sqrt{2} A_{z}=A_{0}+A_{2}, \sqrt{2} A_{\widetilde{z}}=A_{0}-A_{2}, \sqrt{2} A_{w}=A_{1}+A_{3}, \sqrt{2} A_{\widetilde{w}}=A_{1}-A_{3}$ together with $A_{\widetilde{z}}=A_{\widetilde{w}}=0$ implies all gauge fields $A_{\mu}$ must be anti-hermitian. That is, $G=\mathrm{SU}(2)$ gauge theory is realized on $\mathbb{U}_{2}$ successfully.

\section{Comparison to already known soliton solutions}

In this section, we review already known soliton solutions of the anti-self-dual Yang-Mills equation. The four-dimensional complex coordinates $(z, \widetilde{z}, w, \widetilde{w})$ used here is defined as in section 2.1.

\subsection{Atiyah-Ward ansatz solutions $(G=\mathrm{GL}(2))$}

Firstly, we begin with the Atiyah-Ward ansatz solutions [3]. The simplest one is [7]:

$$
J=\left(\begin{array}{cc}
0 & -1 \\
1 & \Delta_{0}
\end{array}\right)
$$

with a scalar function $\Delta_{0}(x)$ and the Yang equation reduces to a simpler linear equation

$$
\left(\partial_{\widetilde{z}} \partial_{z}-\partial_{\widetilde{w}} \partial_{w}\right) \Delta_{0}=0 .
$$

A natural one-soliton solution is given by

$$
\Delta_{0}=\frac{1}{2}\left(e^{L}+e^{-L}\right)=\cosh L, \quad L=(\lambda \beta) z+\alpha \widetilde{z}+(\lambda \alpha) w+\beta \widetilde{w},
$$

and the corresponding action density vanishes: $\operatorname{Tr} F^{2}=0$ by simple calculation. 
The second simplest one is given in the following ansatz:

$$
J=\left(\begin{array}{cc}
\Delta_{0}-\Delta_{1} \Delta_{0}^{-1} \Delta_{-1} & -\Delta_{1} \Delta_{0}^{-1} \\
\Delta_{0}^{-1} \Delta_{-1} & \Delta_{0}^{-1}
\end{array}\right),
$$

which relates to Yang's R-gauge [32] and include the non-linear plane wave solutions in the Minkowski signature [9]. By substituting $J$-matrix into the Yang equation, it reduces to the following chasing equations

$$
\partial_{z} \Delta_{i}=\partial_{\widetilde{w}} \Delta_{i+1}, \quad \partial_{w} \Delta_{i}=\partial_{\widetilde{z}} \Delta_{i+1}
$$

which implies that $\Delta_{0}$ solves the Laplace equation: $\left(\partial_{\widetilde{z}} \partial_{z}-\partial_{\widetilde{w}} \partial_{w}\right) \Delta_{0}=0$.

A natural one-soliton solution is given by

$$
\Delta_{0}=\cosh L, \quad \Delta_{1}=\lambda \cosh L, \quad \Delta_{-1}=\lambda^{-1} \cosh L,
$$

and the corresponding action density is trivial again: $\operatorname{Tr} F^{2}=0$ by simple calculation.

\section{2 't Hooft ansatz solutions $(G=\mathrm{SU}(2))$}

The 't Hooft ansatz [29] (or known as the Corrigan-Fairlie-'t Hooft-Wilczek ansatz [6, 31]) is very important for the study of $G=\mathrm{SU}(2)$ gauge theory on the four-dimensional Euclidean space and is given by

$$
A_{\mu}=i \eta_{\mu \nu}^{(+) a} \sigma_{a} \partial^{\nu} \log \varphi
$$

where $\eta_{\mu \nu}^{(+) a}(a=1,2,3)$ is the self-dual 't Hooft symbol and $\sigma_{a}$ is the Pauli matrices. Under the 't Hooft ansatz, the anti-self-dual Yang-Mills equation reduces to the Laplace equation

$$
\left(\partial_{\bar{z}} \partial_{z}+\partial_{\bar{w}} \partial_{w}\right) \varphi=0
$$

A natural one-soliton solution is given by

$$
\varphi=\frac{1}{2}\left(e^{K}+e^{-K}\right)=\cosh K, \quad K:=k_{\mu} x^{\mu},
$$

where $k_{\mu}$ are real constants which satisfy $k^{2}=k_{\mu} k^{\mu}=0$ due to (4.8). By using some formulas on the 't Hooft symbol, we can easily show that

$$
\operatorname{Tr} F^{2}=-3\left(k^{2}\right)^{2}\left(4 \operatorname{sech}^{4} K-5 \operatorname{sech}^{2} K+2\right) \stackrel{k^{2}=0}{=} 0 .
$$

In conclusion, the action density of the natural one-soliton solutions (4.3), (4.6) and (4.9) are all trivial.

\section{Conclusion and discussion}

In this paper, we constructed exact soliton solutions of four-dimensional anti-self-dual Yang-Mills equations for $G=\mathrm{GL}(2)$ which possess real-valued action densities. Our results showed that such type of solitons can be interpreted as domain wall in four-dimensional 
spaces and $G=\mathrm{U}(2)$ solitons exist on the Ultrahyperbolic signature $\mathbb{U}_{2}$. This fact has a strong connection with $\mathrm{N}=2$ string theories [17, 22, 23].

In $\mathrm{N}=2$ string theories, the equation of motion of the effective action is the Ultrahyperbolic space $\mathbb{U}_{2}$ version of anti-self-dual Yang-Mills equation for $G=\mathrm{U}(2)$. Therefore, our soliton solutions obtained in section 3.4 might give us a hint for finding the corresponding physical objects in these theories. On the other hand, the Euclidean and Minkowski signature version of such kind of $G=\mathrm{U}(2)$ domain wall solutions (or the non-abelian plane waves [4] of the Yang-Mills equation) are still unknown and worth investigating for our future work. These studies might perhaps relate to new perturbative aspects of quantum field theories, new invariants in the four-dimensional geometry or the origin of dark matters someday.

For multi-soliton solutions, we presented these discussions on noncommutative Euclidean spaces explicitly by the noncommutative Darboux transformation in [11] and the noncommutative Bäcklund transformation in [12, 14]. Asymptotic behaviors of these noncommutative soliton solutions were also proved to be the same as in commutative spaces $[11,14]$. It might be an interesting future work to confirm our conjecture that $n$ soliton solutions in $[11,12,14]$ have $n$ isolated localized lumps of energy and preserve their shapes and velocities on each localized solitary wave lump. In addition, explicit analysis of these $n$ soliton scatterings would be expected to give the phase shifts in the scattering processes as discussed in the standard soliton theory (e.g. [19]).

Another interesting problem is to compare the asymptotic behaviors of our solutions $[11,12,14]$ with multi-soliton solutions in [9]. All of these studies might lead to a general formulation of Kodama's Grassmannian approach to the study of soliton scatterings [15], and give a new insight into Hirota's bilinear forms [25] or other formulation of integrable hierarchies $[20,27]$.

\section{Acknowledgments}

$\mathrm{MH}$ is grateful to Jon Nimmo for fruitful discussion and warm hospitality when he visited Glasgow especially in 2006 and 2008. MH also thanks Kanehisa Takasaki for discussion. The work of MH is supported by Grant-in-Aid for Scientific Research (\#16K05318). SCH would like to thank Jon Nimmo and Claire Gilson for private notes and Peiqiang Lin for discussion. The work of SCH is supported by the scholarship of Japan-Taiwan Exchange Association.

\section{A Calculation of action density (2.12)}

\section{Yang's $J$-matrix}

$$
\begin{aligned}
& J=-Q \Lambda^{-1} Q^{-1}=\frac{-1}{\Delta}\left(\begin{array}{cc}
\lambda^{-1} A D-\mu^{-1} B C & \left(\mu^{-1}-\lambda^{-1}\right) A B \\
\left(\lambda^{-1}-\mu^{-1}\right) C D & \mu^{-1} A D-\lambda^{-1} B C
\end{array}\right), \\
& Q=\left(\begin{array}{ll}
A & B \\
C & D
\end{array}\right), \Delta:=\operatorname{det} Q=A D-B C
\end{aligned}
$$




\section{Derivative of $J$-matrix}

$$
J^{\prime}=\frac{\mu^{-1}-\lambda^{-1}}{\Delta^{2}}\left(\begin{array}{cc}
E & F \\
G-E
\end{array}\right) \quad\left\{\begin{array}{l}
E=\left(A C^{\prime}-A^{\prime} C\right) B D-\left(B D^{\prime}-B^{\prime} D\right) A C \\
F=-\left(A C^{\prime}-A^{\prime} C\right) B^{2}+\left(B D^{\prime}-B^{\prime} D\right) A^{2} \\
G=\left(A C^{\prime}-A^{\prime} C\right) D^{2}-\left(B D^{\prime}-B^{\prime} D\right) C^{2}
\end{array}\right.
$$

Gauge field $\left(f^{\prime}:=\partial_{k} f, k=z, w\right)$.

$$
\begin{aligned}
A_{k}= & J^{-1} J^{\prime}=\frac{1}{\Delta^{2}}\left(\begin{array}{cc}
R & S \\
T & -R
\end{array}\right) \\
& \left\{\begin{array}{l}
R=(\mu / \lambda-1)\left(A C^{\prime}-A^{\prime} C\right) B D-(1-\lambda / \mu)\left(B D^{\prime}-B^{\prime} D\right) A C \\
S=-(\mu / \lambda-1)\left(A C^{\prime}-A^{\prime} C\right) B^{2}+(1-\lambda / \mu)\left(B D^{\prime}-B^{\prime} D\right) A^{2} \\
T=(\mu / \lambda-1)\left(A C^{\prime}-A^{\prime} C\right) D^{2}-(1-\lambda / \mu)\left(B D^{\prime}-B^{\prime} D\right) C^{2}
\end{array}\right.
\end{aligned}
$$

Note that if we take $(Q, \Lambda)$ as mentioned in (2.10), then a simple form of $A_{k}$ would be found from the result $A C^{\prime}-A^{\prime} C=2 \lambda p, \quad B D^{\prime}-B^{\prime} D=2 \mu q$ :

$$
\begin{aligned}
A_{k}= & \frac{2(\mu-\lambda)}{\Delta^{2}}\left(\begin{array}{c}
p B D-q A C-p B^{2}+q A^{2} \\
p D^{2}-q C^{2}-p B D+q A C
\end{array}\right) \\
& \left\{\begin{array}{l}
(p, q):=\left(\alpha \varepsilon_{0}, \gamma \widetilde{\varepsilon}_{0}\right) \text { if } m=w, \quad(p, q):=\left(\beta \varepsilon_{0}, \delta \widetilde{\varepsilon}_{0}\right) \text { if } m=z \\
\varepsilon_{0}:=a_{2} c_{1}-a_{1} c_{2}, \quad \widetilde{\varepsilon}_{0}:=b_{2} d_{1}-b_{1} d_{2}
\end{array}\right.
\end{aligned}
$$

Moreover, if we consider the Ultrahyperbolic signature $\mathbb{U}_{2}$ (Take $(Q, \Lambda)$ mentioned in (3.35)), then gauge fields become anti-hermitian naturally:

$$
\begin{aligned}
A_{k}= & \frac{2(\bar{\lambda}-\lambda)}{\Delta^{2}}\left(\begin{array}{c}
p \bar{A} B+\bar{p} A \bar{B}-p B^{2}+\bar{p} A^{2} \\
p \bar{A}^{2}-\bar{p}^{2}-p \bar{A} B-\bar{p} A \bar{B}
\end{array}\right) \\
& \left\{\begin{array}{l}
p:=\alpha \varepsilon_{0}, \quad \text { if } m=w, \quad p:=\beta \varepsilon_{0}, \quad \text { if } m=z, \quad \varepsilon_{0}:=a_{1} \bar{b}_{2}-\bar{a}_{2} b_{1},
\end{array}\right.
\end{aligned}
$$

Field strength $\left(\dot{f}:=\partial_{l} f, l=\widetilde{z}, \widetilde{w}\right)$

$$
\begin{aligned}
F_{k l}= & -\partial_{l} A_{k}=\frac{2(\lambda-\mu)}{\Delta^{2}}\left(\begin{array}{cc}
U & V \\
W & -U
\end{array}\right) \\
& \left\{\begin{array}{l}
U=p[\dot{B} D+B \dot{D}-2 B D(\dot{\Delta} / \Delta)]-q[\dot{A} C+A \dot{C}-2 A C(\dot{\Delta} / \Delta)] \\
V=-2 p\left[B \dot{B}-B^{2}(\dot{\Delta} / \Delta)\right]+2 q\left[A \dot{A}-A^{2}(\dot{\Delta} / \Delta)\right] \\
W=2 p\left[D \dot{D}-D^{2}(\dot{\Delta} / \Delta)\right]-2 q\left[C \dot{C}-C^{2}(\dot{\Delta} / \Delta)\right]
\end{array}\right.
\end{aligned}
$$

Note that $p, q$ are defined as in (A.5) and $A_{l}=0$ as mentioned in (2.6). 


\section{Action density}

$$
\begin{aligned}
& \operatorname{Tr} F_{w \widetilde{z}} F_{z \widetilde{w}}=\frac{16(\lambda-\mu)^{2} \varepsilon_{0} \widetilde{\varepsilon}_{0}}{\Delta^{4}}\left\{4 \varepsilon_{0} \widetilde{\varepsilon}_{0} \alpha \beta \gamma \delta+(\alpha \delta-\beta \gamma)^{2}(A \dot{D}-\dot{B} C)(\dot{A} D-B \dot{C})\right. \\
& +\alpha \beta \gamma \delta[(A D-B C)(\dot{A} \dot{D}-\dot{B} \dot{C})+(\dot{A} D-B \dot{C})(A \dot{D}-\dot{B} C)]\}, \\
& \operatorname{Tr} F_{w \widetilde{w}}^{2}=\frac{16(\lambda-\mu)^{2} \varepsilon_{0} \widetilde{\varepsilon}_{0}}{\Delta^{4}}\left\{2 \varepsilon_{0} \widetilde{\varepsilon}_{0}\left(\alpha^{2} \delta^{2}+\beta^{2} \gamma^{2}\right)+\right. \\
& +\alpha \beta \gamma \delta[(A D-B C)(\dot{A} \dot{D}-\dot{B} \dot{C})+(\dot{A} D-B \dot{C})(A \dot{D}-\dot{B} C)]\}, \\
& \operatorname{Tr} F^{2}=\operatorname{Tr} F_{m n} F^{m n}=4\left(\operatorname{Tr} F_{w \widetilde{z}} F_{z \widetilde{w}}-\operatorname{Tr} F_{w \widetilde{w}}^{2}\right) \\
& =\frac{64(\lambda-\mu)^{2}(\alpha \delta-\beta \gamma)^{2} \varepsilon_{0} \widetilde{\varepsilon}_{0}}{\Delta^{4}}\left[(A \dot{D}-\dot{B} C)(\dot{A} D-B \dot{C})-2 \varepsilon_{0} \widetilde{\varepsilon}_{0}\right]
\end{aligned}
$$

Finally, substituting (2.10) into the above formula, we get (2.12).

Open Access. This article is distributed under the terms of the Creative Commons Attribution License (CC-BY 4.0), which permits any use, distribution and reproduction in any medium, provided the original author(s) and source are credited.

\section{References}

[1] A. Actor, Classical Solutions of SU(2) Yang-Mills Theories, Rev. Mod. Phys. 51 (1979) 461 [INSPIRE].

[2] M.F. Atiyah, N.J. Hitchin, V.G. Drinfeld and Y. Manin, Construction of Instantons, Phys. Lett. A 65 (1978) 185 [INSPIRE].

[3] M.F. Atiyah and R.S. Ward, Instantons and Algebraic Geometry, Commun. Math. Phys. 55 (1977) 117 [INSPIRE].

[4] S.R. Coleman, Nonabelian Plane Waves, Phys. Lett. B 70 (1977) 59 [InSPIRE].

[5] S. Coleman, Aspects of Symmetry, Cambridge University Press, (1985).

[6] E. Corrigan and D.B. Fairlie, Scalar Field Theory and Exact Solutions to a Classical SU(2) Gauge Theory, Phys. Lett. B 67 (1977) 69 [InSPIRE].

[7] E. Corrigan, D.B. Fairlie, R.G. Yates and P. Goddard, The Construction of Selfdual Solutions to SU(2) Gauge Theory, Commun. Math. Phys. 58 (1978) 223 [InSPIRE].

[8] N.S. Craigie, P. Goddard and W. Nahm, Monopoles in Quantum Field Theory, World Scientific, (1982).

[9] H.J. de Vega, Nonlinear Multiplane Wave Solutions of Selfdual \{Yang-Mills\} Theory, Commun. Math. Phys. 116 (1988) 659 [INSPIRE].

[10] N. Dorey, T.J. Hollowood, V.V. Khoze and M.P. Mattis, The Calculus of many instantons, Phys. Rept. 371 (2002) 231 [hep-th/0206063] [inSPIRE].

[11] C.R. Gilson, M. Hamanaka, S.-C. Huang and J.J.C. Nimmo, Soliton Solutions of Noncommutative Anti-Self-Dual Yang-Mills Equations, J. Phys. A 53 (2020) 404002 [arXiv: 2004.01718] [INSPIRE].

[12] C.R. Gilson, M. Hamanaka and J.J.C. Nimmo, Bäcklund Transformations and the Atiyah-Ward ansatz for Noncommutative Anti-Self-Dual Yang-Mills Equations, Proc. Roy. Soc. Lond. A 465 (2009) 2613 [arXiv:0812.1222] [INSPIRE]. 
[13] D.J. Gross, R.D. Pisarski and L.G. Yaffe, $Q C D$ and Instantons at Finite Temperature, Rev. Mod. Phys. 53 (1981) 43 [INSPIRE].

[14] M. Hamanaka and H. Okabe, Soliton Scattering in Noncommutative Spaces, Theor. Math. Phys. 197 (2018) 1451 [Teor. Mat. Fiz. 197 (2018) 68] [arXiv:1806.05188] [INSPIRE].

[15] Y. Kodama, KP Solitons and the Grassmannians, Springer, (2017).

[16] N.S. Manton and P. Sutcliffe, Topological solitons, Cambridge University Press, (2004).

[17] N. Marcus, The N=2 open string, Nucl. Phys. B 387 (1992) 263 [hep-th/9207024] [INSPIRE].

[18] L.J. Mason and N.M. Woodhouse, Integrability, Self-Duality, and Twistor Theory, Oxford University Press, (1996).

[19] V.B. Matveev and M.A. Salle, Darboux Transformations and Solitons, Springer-Verlag, (1991).

[20] Y. Nakamura, Transformation group acting on a self-dual Yang-Mills hierarchy, J. Math. Phys. 29 (1988) 244 [INSPIRE].

[21] J.J.C. Nimmo, C.R. Gilson and Y. Ohta, Applications of Darboux transformations to the selfdual Yang-Mills equations, Theor. Math. Phys. 122 (2000) 239 [Teor. Mat. Fiz. 122 (2000) 284] [INSPIRE].

[22] H. Ooguri and C. Vafa, Geometry of $N=2$ strings, Nucl. Phys. B 361 (1991) 469 [InSPIRE].

[23] H. Ooguri and C. Vafa, $N=2$ heterotic strings, Nucl. Phys. B 367 (1991) 83 [InSPIRE].

[24] A.M. Polyakov, Gauge Fields and Strings, Harwood Academic, (1987).

[25] N. Sasa, Y. Ohta and J. Matsukidaira, Bilinear Form Approach to the Self-Dual Yang-Mills Equation and Integrable System in (2+1)-Dimension, J. Phys. Soc. Jap. 67 (1998) 83.

[26] M.A. Shifman, Instantons in Gauge Theories, World Scientific, (1994).

[27] K. Takasaki, A New Approach To The Selfdual Yang-Mills Equations, Commun. Math. Phys. 94 (1984) 35 [INSPIRE].

[28] G. 't Hooft, unpublished.

[29] G. 't Hooft, 50 Years of Yang-Mills Theory, World Scientific, (2005).

[30] R.S. Ward, Integrable and solvable systems, and relations among them, Phil. Trans. Roy. Soc. Lond. A 315 (1985) 451.

[31] F. Wilczek, Geometry and interactions of instantons, in Quark Confinement and Field Theory, Wiley, (1977), pg. 211.

[32] C.N. Yang, Condition of Selfduality for SU(2) Gauge Fields on Euclidean Four-Dimensional Space, Phys. Rev. Lett. 38 (1977) 1377 [INSPIRE]. 